\title{
An Application of RFID in Monitoring Agricultural Material Products
}

\author{
Jianhui $\mathrm{Du}^{1,2}$, Peipei $\mathrm{Li}^{1}$, Wanlin Gao ${ }^{1,3, *}$, Dezhong Wang ${ }^{1}$, Qing Wang ${ }^{1}$, \\ and Yilong $\mathrm{Zhu}^{1}$ \\ ${ }^{1}$ College of Information and Electrical Engineering, China Agricultural University, \\ Beijing, P.R. China 100083 \\ ${ }^{2}$ Yunnan Agriculture Department, Yunnan Province, P.R. China 650224 \\ ${ }^{3}$ College of Information and Electrical Engineering, China Agricultural University, \\ No. 17, Qinghua Dong Lu, Haidian, Beijing 100083, P.R. China, \\ Tel.: +86-010-62736755; Fax: +86-010-62736755 \\ gaowlinecau.edu.cn
}

\begin{abstract}
With the development of modern agriculture, more and more agricultural material products are used in it. While how to keep these things safe is a big problem at present, which needs to be paid more attention. This article develops an agricultural material products monitor system based on RFID which gives alarm as soon as possible if there is anything unmoral. Every warehouse exit is equipped with a RFID reader, while each agricultural material product has a tag on them. When passing though, the reader identifies the tag's information and transfer it to the PC, The PC inquiries the database storing all tags' information, and tells which one is not taken out legally by alarming aloud.
\end{abstract}

Keywords: agricultural material products, RFID, monitor, reader, tag, alarm.

\section{Introduction}

Nowadays with socio-economic development, based on abundant agricultural resources, the agriculture of our country has been developing a lot, more and more agricultural material products are used in it, such as fertilizers, pesticides and so on. However this has brought much pressure to daily management and many problems occur at the same time. Products theft is one of these problems, which does cause properties loss and hurt the owners in psychology. It is necessary for us to find a way to monitor agricultural material products from being taken out of the warehouse by thefts.

Usually we take products supervision with cameras, which can't tell whether the things are taken out of the warehouse legally or not in real time. When the truth is found, they are already in miles away. Or the thieves may hide the things when pass by the camera to avoid exposure. This paper aims to develop a real time monitoring system based on RFID (radio frequency identification) which is widely used in Warehouse custody, access control, highway inspection, rail inspection, retail stores, libraries and many other areas.

\footnotetext{
* Corresponding author.
} 


\section{RFID System}

RFID (Radio Frequency Identification) is a non-touch automatic identification technology rising in 1990s. In the early information period, manual data-input and identification brought a large number of problems such as labor-intensive, high bit error rate and lack of real-time, etc. In order to solve these problems, people developed many kinds of identification technologies such as bar code, magnetic card, IC card, biometrics, voice recognition and wireless radio frequency identification, which are very common in our present life. RFID is one of the most promising means which makes use of electromagnetic or inductive coupling between the reader and the tag adhesive to objects for data communications, automatic target recognition and retrieve relevant data without human intervention(Dingyi Dai et al., 2006; Automatic Identification Manufacture Association of China., 2003).

A complete RFID system consists of three parts: the transponder (tag), reader, as well as back-end database. The radio frequency identification includes tag and reader (Zhanqing You et al., 2005).

Tag: consists of coupling components and chips. Each electronic tag is attached to the target object and has a global unique identifier (ID) preserving electronic data in prescribe format, which can not be changed or fabricate. In practical applications, tags are attached to the surface of the objects to be identified. According to the power supply, tags can be classified into active tags and passive ones. As the name suggest, active tags means the tag has power (i.e. batteries) inside, while passive tags do not have.

Reader: an equipment for reading (or writing) RFID information, which can be hand-held or fixed. Reader can identify the preserved electronic data given by RFID tags so as to achieve the purpose of automatic identification of objects. Reader is usually connected to the computer and sends the recognized information to the computer for the next process.

Electronic tags and readers complete spatial couple of RF signals (non-touch) through the coupling components (antenna) for successful energy transfer and data exchange on the coupling channel according to temporal relations. There are two types of radio frequency signals between reader and tag: inductive coupling and electromagnetic backscatter coupling. The former achieves coupling through the spatial highfrequency alternating magnetic field, based on the law of electromagnetic induction; the latter model is based on the principle of radar: when electromagnetic wave is launched out, touched the target and reflected, it will bring back the target information.

Inductive coupling manner is suitable for the intermediate frequency and lowfrequency radio frequency identification system at close range. Typical operating frequency include: $125 \mathrm{kHz}, 225 \mathrm{kHz}$ and $13.56 \mathrm{MHz}$. Distance identified is less than $1 \mathrm{~m}$, typically $10 \mathrm{~cm} \sim 20 \mathrm{~cm} .13 .56 \mathrm{MHz}$ frequency band is widely used in public traffic card.

Electromagnetic backscatter coupling is generally suitable for high frequency, ultra-high frequency, as well as microwave radio frequency identification systems for long distance. Ultra-high frequency (UHF) band radio frequency and microwave tags are referred as microwave radio frequency tags with the typical operating frequency is: $433.92 \mathrm{MHz}, 862(902) \sim 928 \mathrm{MHz}, 2.45 \mathrm{GHz}, 5.8 \mathrm{GHz}$. The RF tag reader antenna is located within the far zone radiation field of the reader antenna. Coupling between 
tags and readers is electromagnetic. Reader antenna radiation field provides energy for passive radio frequency tags and wakes up the active tags. The corresponding distance is generally greater than $1 \mathrm{~m}$, typically $4 \sim 6 \mathrm{~m}$, up to longer than $10 \mathrm{~m}$ (Xuezong Tao et al., 2006; Xiaoguang Zhou et al., 2006).

Based on current technological level, the relatively successful passive microwave RF products work mainly on $902 \sim 928 \mathrm{MHz}$ frequency bands. $2.45 \mathrm{GHz}$ and $5.8 \mathrm{GHz}$ radio frequency identification system are more available with the semi-passive microwave RFID products. Semi-passive tags are generally button battery powered, with a relatively long reading distance. Typical characteristics of a microwave RF tag are mainly concentrated on passive or active, wireless reading-writing distance, whether to support the multi-tag reading and writing, whether to suitable for the highspeed applications identification, the reader's transmission power tolerance, prices of tags and reader, etc. Typical applications of microwave radio frequency tag include: mobile vehicle identification, electronic ID cards, storage and logistics applications, electronic anti-theft locking (electronic controller for remote control door) and so on.

In general, RFID works as follows: tags enter the magnetic field; receive signals emitted by the reader; send the product information stored in the chip through the induced currents obtained by the energy (Passive Tag), or send a frequency signal actively (Active Tag); readers read the information and decoded; send the information to the central system to be processed. The basic model of Radio frequency identification system is shown as in Fig.1.

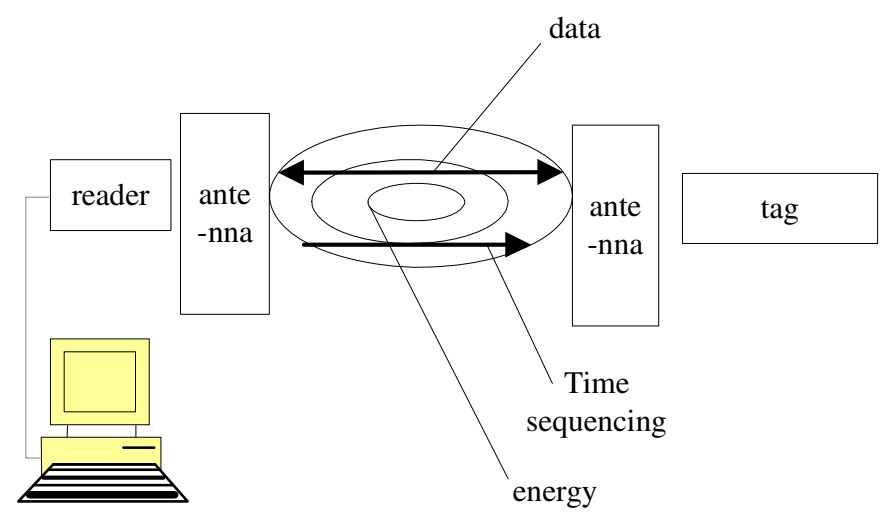

Fig. 1. The basic model of radio frequency identification system

\section{Warehouse Agricultural Material Products Monitoring System}

In the warehouse products monitoring system, the owner and their agricultural material products are equipped with a unique ID card each. The radio frequency readers which are connected with the PC are equipped at each warehouse exit. The PC has a backstage database for the monitoring system keeping tag information of all the owners and products. If the valuable is taken out by someone who is not the owner, the system will alarm, thus rises the owners' attention and achieves timely inspection. 
The working process of system is explained as follows: When someone leaves the warehouse, the reader reads the cards' information. If there are label information of human and products identified simultaneously, check if the human information is legal(correspond to the database information) and gives warning or not; If only products information, but nobody's information is identified, that means a non-owner carries over the products, the system alarms.

Supposes all the owners' card number is A1/A2/A3..., the products cards are numbered as $\mathrm{B} 1, \mathrm{~B} 2 \ldots$ respectively. If the system identifies $\mathrm{A} 1 / \mathrm{A} 2 \ldots$ and $\mathrm{B} 1 / \mathrm{B} 2 \ldots$ (one or several) at the same time (possible have difference of some amount of time, like several seconds), it does not alarm; If $\mathrm{A} 1 / \mathrm{A} 2 \ldots$ but not $\mathrm{B} 1 / \mathrm{B} 2 / \ldots$ (one or several) is identified, it gives no alarm; If $\mathrm{B} 1 / \mathrm{B} 2 / \ldots$ but without $\mathrm{A} 1 / \mathrm{A} 2 \ldots$ (one or several), it alarms. Fig.2. shows the hardware configuration of monitoring system.

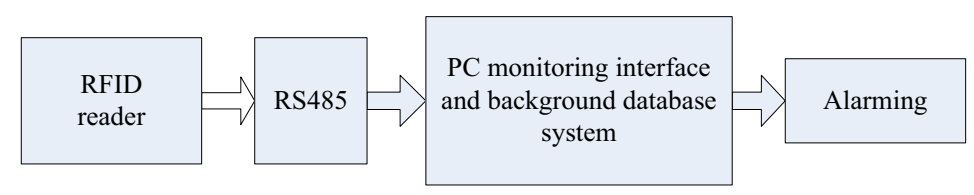

Fig. 2. Hardware configuration of monitoring system

This system is based on the prerequisite of that all owners and their products have been equipped with a tag and their information are stored in the database, Fig.3. shows the flow chart of this system.

Multi-tag reading technology involved in this system is a key point. It is different from the access control system or traffic card system we usually see, which can only identify one card each time. This monitoring system involves issues of legality of people and products, and when products are brought out and people walk out, their information must match. The system can identify a number of tags at same time or within a short period of time (seconds). Moreover, system should be passive to reduce energy loss and save the cost of tags.

According to the introduction to the RFID systems, as well as the actual needs, the system uses $920-925 \mathrm{MHz}$ ultra-high frequency section for the identification system and passive UHF RFID tags with corresponding standard as the system tags. The system uses U-CODE GEN2 card, which operates on $860-960 \mathrm{MHz}$, following ISO 18000-6C standards and EPC C1 Gen2 standard, with 512 bit memory, readingwriting distance of up to $3-10 \mathrm{~m}$ (with the distribution of the antenna). It is a readingwriting card and can work at the speed of up to $60 \mathrm{~km} / \mathrm{h}$, suitable for the rapid identification. It has anti-collision mechanism, suitable for multi-tag reading.

S1871 is a short-range multi-purpose mini-UHF reader module. It is based on the Intel R1000 micro UHF reader module and can be embedded in fixed or mobile devices. Power range is in line with the mainstream standards such as European (ETSI EN 302 208) and U.S. (FCC part 15). It's adjustable with maximum power of $1300 \mathrm{~mW}$, supporting EPC Class1 Gen2 protocol and using dipole antenna(RFID World Forum, 2007). 


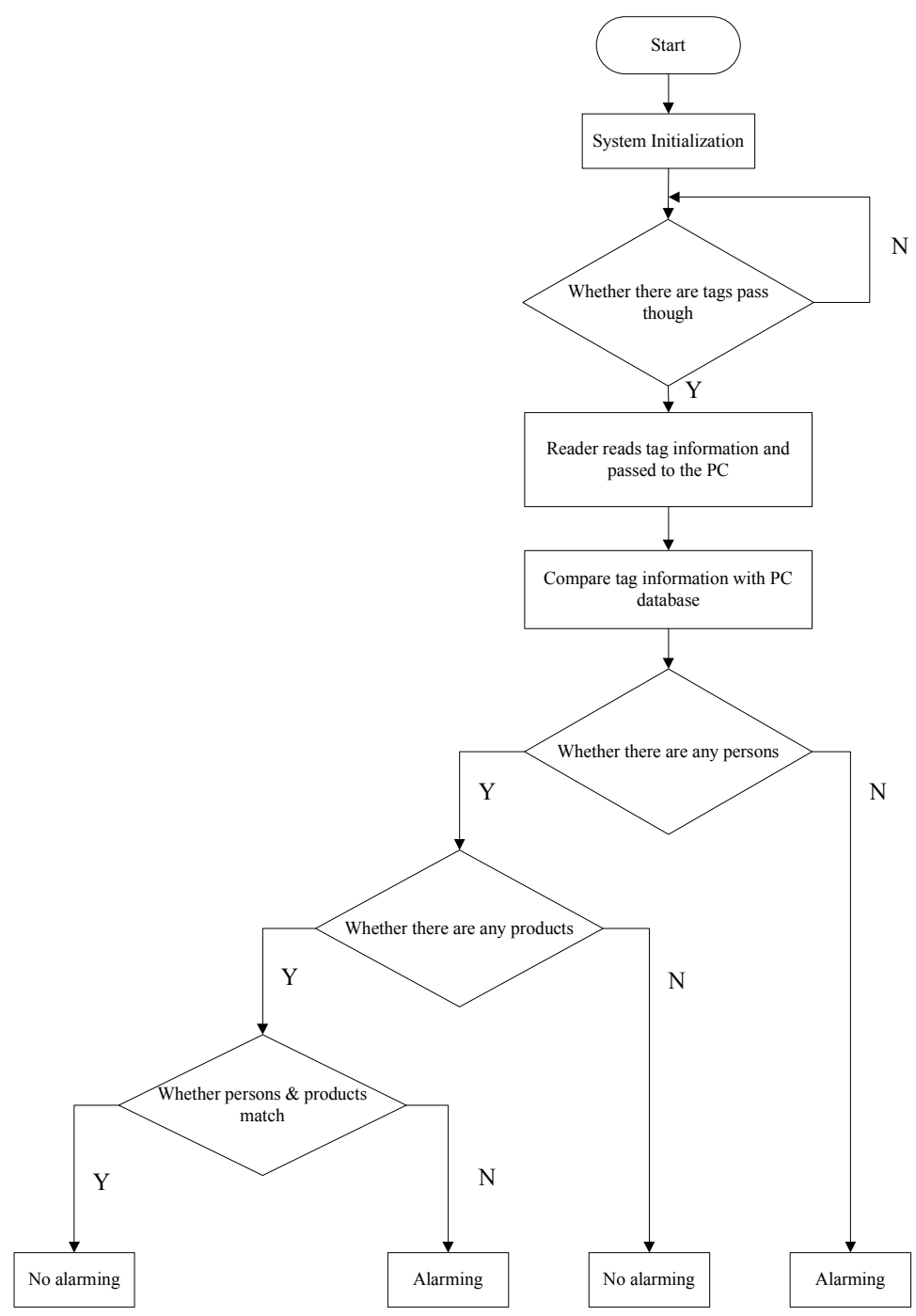

Fig. 3. Warehouse products monitoring system work flow chart

The system uses 920-925MHz UHF frequency band, following the latest ISO / IEC, ISO 18000-6C and EPC global organization, EPC C1 Gen2. The usage of these standards makes preparation for the idea of the Global objects of great networking in future. Moreover, since it is passive system, and can identify objects within a long distance, it is widely used in many areas, such as high-speed train system which are familiar to us(Embedded online., 2007).

Communications between PC and reader are achieved through the serial port, generally RS232 and RS485(Chaoqing Li et al., 2002). The system adopts RS485 for it working relatively within a long distance. PC software is developed with Visual Studio2005, using SerialPortComm to achieve serial communication. Access database 
in PC has all information of owners and their products. The program can receive and store tags' information at real-time, query database and display the corresponding information through graphic interface. (Weichen Lv et al., 2006; Qiming studio., 2004).

When tag information is identified, the system will test if it is owners' or products' or both. Then:

Only owners', no alarming;

Only products', alarming;

If information of owners and products are both identified, the system queries the owners' tag information in the database to tell who is passing; then queries the products' tag information in to know whose products they are, According to the result , the system alarms or not.

\section{Conclusion}

1. The valuables monitoring system based on RFID technology, using UHF frequency band, supports accuracy identification and multi-passive-tags to meet the monitoring requirements completely, and therefore achieves the real-time and accuracy.

2. We have applied this system to monitor the warehouse agricultural material products in the real life, the correct recognition rate could be up to $99.5 \%$, which meet our demand primely.

3. The system follows the most advanced international standards and can be widely applied in future.

\section{References}

Automatic Identification Manufacture Association of China. Barcodes and RFID application note. Mechanical Industry Press, Beijing (2003)

Li, C.: PC machine and single-chip data communications technology. Beijing University of Aeronautics and Astronautics Press, Beijing (2002)

Dai, D.: RFID technology in China Development. RFID technology and application (2006)

Embedded online. Abroad RFID: expand the functions of the application to broaden the growing popularity [R/OL] (2007), http: / / www . mcuol.com/News / 147/8105.htm

Qiming studio. Visual C++ + SQL Server database application system development and examples. Posts \& Telecom Press, Beijing (2004)

Lv, W., Huo, Y., Lv, B., et al.: Primary and increased Visual C \#. Tsinghua University Press, Beijing (2006)

Zhou, X.: Radio Frequency Identification (RFID) technology and application. Posts \& Telecom Press, Beijing (2006)

Tao, X.: China's RFID technology analysis. RFID technology and application (2006)

RFID World Forum. Sense UHF RFID reader module for micro embedded [R/OL] (2007), http: / / www.rfidworld.com.cn/bbs / disptopic . asp?boardid=56\&topi cid $=8497$

You, Z., Li, S.: Radio Frequency Identification Technology Theory and Application. Publishing House of Electronics Industry, Beijing (2005) 